\title{
Factores asociados a la presencia de prostatitis aguda bacteriana en pacientes sometidos a biopsia prostática transrectal entre los años 2015 y 2019 en un hospital de las Fuerzas Armadas de Lima, Perú
}

\section{Factors associated with the presence of acute bacterial prostatitis in patients undergoing transrectal prostate biopsy between 2015 and 2019 at an Armed Forces Hospital in Lima, Peru}

Correspondencia Jorge Luis Maguiña Quispe jmaguinaq@ucientifica.edu.pe

Recibido: 21/02/2020

Arbitrado por pares

Aprobado: 25/03/2020

Citar como: Collado-Mendiola S, Lenz-Lee T, Alban-Moral SD, Maguiña JL. Factores asociados a la presencia de prostatitis aguda bacteriana en pacientes sometidos a biopsia prostática transrectal entre los años 2015 y 2019 en un Hospital de las Fuerzas Armadas de Lima, Perú. Acta Med Peru. 2020:37(1):34-9. doi: https://doi. org/10.35663/amp.2020.371.891
Stefanie Collado-Mendiola ${ }^{1 a}$, Tiziana Lenz-Lee ${ }^{1 a}$, Sergio D. Alban-Moral²b, Jorge L. Maguiña ${ }^{1 c}$

1 Facultad de Ciencias de la Salud, Universidad Científica del Sur. Lima, Perú.

2 Servicio de Urología, Hospital Militar Central "Coronel Luis Arias Schreiber". Lima, Perú.

Médico cirujano, ${ }^{\mathrm{b}}$ médico urólogo, ${ }^{\mathrm{c}}$ tecnólogo médico

\section{RESUMEN}

Objetivo: determinar los factores asociados a la presencia de prostatitis aguda bacteriana en pacientes sometidos a biopsia prostática transrectal entre los años 2015 y 2019 en un Hospital de las Fuerzas Armadas de Lima, Perú. Materiales y métodos: estudio observacional transversal retrospectivo. Se evaluaron historias clínicas de 142 pacientes, seleccionados por muestreo no probabilístico. Analizamos los factores asociados a la presencia de prostatitis aguda bacteriana $(\mathrm{PAB})$ en pacientes sometidos a biopsia prostática transrectal (modelo-1) y el número de factores asociados a PAB (modelo-2). Resultados: la presencia de prostatitis aguda bacteriana (PAB) fue $15,5 \%$. La PAB fue mayor en pacientes diabéticos, hipertensos y los que padecían de insuficiencia renal crónica. En el modelo-1, el antecedente de diabetes mellitus tipo 2 (razón de prevalencia ajustada [RPa]: 3,18; IC 95\%: 0,92 - 11,03), la hipertensión arterial (RPa: 1,78; IC 95\%: 0,53 5,98 ), la insuficiencia renal (RPa: 2,73; IC 95\%: 0,60-12,46) y la prostatitis crónica (RPa: 4,5; IC $95 \%: 0,99-20,43)$ no se encontraron asociados $(p>0,05)$. En el modelo-2, encontramos que aquellos sujetos entre 66-75 años, tienen $50 \%$ menor probabilidad de presentar PAB que aquellos sujetos de 50-65 años y que los sujetos mayores de 75 años tienen 2,7 (IC 95\%: 0,86-8,13) veces mayor probabilidad que el grupo más joven. Finalmente, se observó que un mayor número de comorbilidades incrementa la probabilidad de presentar PAB, siendo 4,80 (IC 95\%: 1,86-12,42) veces en aquellos con una enfermedad, 5,77 (IC 95\%: 1,03-32,13) con dos enfermedades y 10,47 (IC 95\%: 1,98 - 55,41) veces mayor, en aquellos con tres enfermedades. Conclusiones: en nuestro estudio, el antecedente de diabetes mellitus tipo 2 y el número de comorbilidades crónicas incrementan la probabilidad de presentar PAB. Se recomienda realizar un mayor número de estudios que permitan verificar nuestros resultados.

Palabras clave: Prostatitis aguda bacteriana; Biopsia; Diabetes mellitus; Hipertensión (fuente: DeCS-BIREME). 


\begin{abstract}
Objective: to determine the factors associated with the presence of acute bacterial prostatitis in patients undergoing transrectal prostate biopsy between 2015 and 2019 at an Army Forces Hospital in Lima, Peru. Materials and methods: this is a retrospective observational and cross-sectional study. Clinical records from 142 patients were reviewed, which were selected using non-probabilistic sampling. We analyzed factors associated with the occurrence of acute bacterial prostatitis (ABP) in patients undergoing a transrectal prostate biopsy (model 1), and the number of factors associated with ABP (model 2). Results: the rate of ABP was $15.5 \%$. This condition was more prevalent in subjects with diabetes, high blood pressure and chronic renal failure. In model 1, a past history of type 2 diabetes mellitus (adjusted prevalence ratio [aPR]: 3.18; 95\% Cl: 0.92-11.03), high blood pressure (aPR: 1.78; 95\% Cl: 0.53-5.98), renal failure (aPR: $2.73 ; 95 \% \mathrm{Cl}: 0.60-12.46$ ), and chronic prostatitis (aPR: $4.5 ; 95 \% \mathrm{Cl}: 0.99-20.43$ ) were not associated ( $p>0.05$ ). In model 2 , we found that subjects between $66-75$ years of age had a $50 \%$ less likelihood for developing APB compared with those in the 50-65 years old group, and that subjects more than 75 years old have a 2.7 -fold $(95 \% \mathrm{Cl}: 0.86-8.13$ ) likelihood for this condition compared with subjects from younger age groups. Finally, it was observed that a greater number of comorbidities increases the likelihood for developing $A B P$, being this 4.80 -fold $(95 \% \mathrm{Cl}: 1.86-12.42)$ in those with one concomitant disease, 5.77 -fold (95\% $\mathrm{Cl}: 1.03-32.13)$ in those with two concomitant diseases, and 10.47 - fold ( $95 \% \mathrm{Cl}: 1.98-55.41)$ in those who had three concomitant diseases. Conclusions: in this study, a past history of type 2 diabetes mellitus and the number of concomitant chronic conditions increase the likelihood for developing ABP. It is recommended to perform further research on this topic, so our results may be verified.
\end{abstract}

Keywords: Acute bacterial prostatitis; Biopsy; Diabetes mellitus; Hypertension (source: MeSH NLM).

\section{INTRODUCCIÓN}

La prostatitis aguda bacteriana (PAB) constituye uno de los síndromes prostáticos causantes de sintomatología urinaria y dolor pélvico en varones ${ }^{[1]}$. Ocurre luego de una biopsia prostática y es causada debido a que los microorganismos penetran el tejido prostático mediante la inoculación directa de bacterias de la mucosa rectal por la aguja de biopsia ${ }^{[2]}$. Las bacterias aisladas con mayor frecuencia en esta cuadro son los bacilos gramnegativos, siendo el $90 \%$ de los casos Escherichia coli y el resto Klebsiella, Pseudomonas y otros cocos grampositivos como: Enterococcus, Streptococcus y Staphylococcus ${ }^{[3]}$.

Clínicamente, la PAB está caracterizada por la presencia de dolor a nivel suprapúbico o rectal, acompañada de síntomas sistémicos (escalofríos, fiebre, náuseas y vómitos) y síntomas urinarios bajos (disuria, polaquiuria, urgencia). De todos estos, los más frecuentes son la fiebre y la disuria, presentes en más de la mitad de los pacientes. La hematuria puede observarse en algunos casos entre el 17 al $20 \%$ de los pacientes ${ }^{[4]}$.

La biopsia prostática transrectal eco-dirigida es un procedimiento mínimamente invasivo y es en la actualidad el estándar de oro para la obtención de muestras que corroboran el diagnóstico de cáncer de próstata ${ }^{[5]}$. Es considerada un procedimiento seguro y confiable pero no exento de complicaciones, las cuales incluyen bacteriuria asintomática, infecciones del tracto urinario, prostatitis, bacteriemia e incluso sepsis ${ }^{[6]}$. La necesidad de realizar una biopsia prostática está determinada típicamente por los niveles séricos del antígeno prostático específico (PSA) y/o un tacto rectal sospechoso durante el examen físico del paciente ${ }^{[3]}$.

Las complicaciones infecciosas de la biopsia prostática transrectal han aumentado en los últimos años. Según la Asociación Americana de Urología, las frecuencias de infección reportadas varían de un 0,1 a 7,0\% y de las cifras anteriormente descritas, un 2,0 a $6,0 \%$ de los casos corresponden a infecciones del tracto urinario y prostatitis, de los cuales 30 a $50 \%$ además contraen bacteriemia, y pueden llegar a desarrollar sepsis ${ }^{[7]}$. Existen además factores asociados que contribuyen a la presencia de $\mathrm{PAB}$, como la presencia de diabetes, prostatitis crónica e inmunosupresión ${ }^{[8]}$. Estos factores, propios de cada paciente, así como la edad y el uso de sonda Foley podrían incrementar el riesgo de una persona, que será sometida a una biopsia transrectal, de padecer PAB.

Los factores de riesgo para el desarrollo de complicaciones infecciosas después de una biopsia prostática transrectal siguen siendo controversiales. Varios estudios han demostrado que la hospitalización reciente, las comorbilidades (particularmente diabetes), antecedentes de infecciones urinarias y antecedentes de uso de antibióticos se asociaron significativamente con mayor frecuencia de complicaciones infecciosas ${ }^{[9]}$. No obstante, en el Perú, hasta la fecha no existen estudios previos que hayan reportado la frecuencia de complicaciones infecciosas o no infecciosas después de una biopsia prostática transrectal. Por lo tanto, nosotros nos propusimos evaluar los factores asociados a la presencia de prostatitis aguda bacteriana en pacientes sometidos a una biopsia prostática transrectal.

\section{MATERIALES Y MÉTODOS}

\section{Diseño y población de estudio}

Estudio observacional, transversal, analítico y retrospectivo. El estudio fue realizado en el Hospital Militar Central Coronel Luis Arias Schreiber de Lima - Perú, en el cual la gran mayoría de población atendida es personal del ejército peruano, durante los meses de octubre y diciembre del 2019. Se consideró como 
unidad de análisis a las historias clínicas de todos los pacientes varones sometidos a biopsia prostática transrectal desde enero del año 2015 hasta marzo del año 2019.

\section{Cálculo del poder estadístico}

Se realizó en el software libre OpenEpi ${ }^{[10]}$. Nosotros obtuvimos un poder estadístico menor al $80 \%$ para un total de 142 sujetos mayores de 50 años, una frecuencia esperada de diabetes del $10 \%$ y una razón de prevalencia de 1,42, basado en los resultados obtenidos por Emanuel Repetto et al. ${ }^{[8]}$. El número de pacientes que fueron sometidos a biopsia prostática transrectal entre el 2015 y el 2019 fueron 170, de los cuales solo 142 historias clínicas presentaron datos completos para nuestro análisis.

\section{Criterios de elegibilidad}

Se incluyeron todas las historias clínicas de hombres sometidos a biopsia prostática transrectal en el periodo de tiempo mencionado, y que contaban con una evaluación urológica previa, un tacto rectal positivo, una profilaxis antibiótica adecuada y el uso de enema evacuante previo al procedimiento.

Se excluyeron las historias clínicas de aquellos pacientes que contaban con un urocultivo positivo previo al procedimiento, al igual que pacientes con registros de infecciones urinarias a repetición y aquellos registros con información incompleta.

\section{Variables}

La variable resultado en el estudio fue la presencia o no de un diagnóstico de prostatitis aguda bacteriana y/o infección del tracto urinario posterior a una biopsia transrectal por historia clínica. Las otras covariables evaluadas, fueron la edad (categorizada en: $50-65,66-75,>75$ años), el diagnóstico previo de diabetes mellitus tipo 2 (sí/no), hipertensión arterial (sí/no), insuficiencia renal crónica (sí/no), infarto agudo al miocardio (sí/no), accidente cerebrovascular (sí/no), prostatitis crónica (sí/no), entre otros factores como glaucoma, hipotiroidismo, artrosis, gastritis.

Además, para efectos del análisis, construimos la variable número de enfermedades crónicas; la cual se definió como la presencia de uno, dos, tres o ninguna de las siguientes enfermedades crónicas evaluadas en el estudio: diabetes mellitus tipo 2, hipertensión arterial e insuficiencia renal.

\section{Procedimiento de recolección de datos}

Se revisaron las historias clínicas provenientes del archivo del hospital en el período de tiempo de octubre a diciembre del 2019 para buscar los registros de pacientes sometidos a biopsia prostática transrectal y valorar, mediante la ficha de recolección de datos elaborada, los factores de riesgo asociados al desarrollo de prostatitis aguda bacteriana.

El hospital, ha adaptado las recomendaciones de la Asociación Americana de Urología y las ha incorporado como parte de los protocolos de diagnóstico de PAB. Por lo tanto, su diagnóstico se basa en la presencia de fiebre con picos intermitentes, dificultad al miccionar, dolor perineal, presencia de orina turbia y el incremento del tamaño de la próstata en su evaluación al tacto rectal. Los exámenes complementarios de laboratorio incluyen la evaluación de leucocitosis en el hemograma, presencia de leucocitos en la secreción prostática y un urocultivo o hemocultivo positivo.

Todos los datos de las historias clínicas fueron ingresados a una ficha de recolección de datos, creada para este propósito, para luego ser digitadas en una base de datos de Microsoft Excel ${ }^{\circledR}$ por doble digitación como criterio de control de calidad.

\section{Análisis estadístico}

Todos los análisis fueron realizados en Stata versión 14,0 (StataCorp, College Station, TX, USA) y se consideró como significativo un valor de $p$ menor a 0,05.

El análisis univariado de las variables cuantitativas incluyo el cálculo de medidas de tendencia central y dispersión. En el caso de las variables cualitativas, se incluyeron cálculos de frecuencias absolutas y relativas. Para el análisis bivariado se realizó la prueba de Chi-cuadrado de Pearson o la prueba Exacta de Fisher. Finalmente, para el cálculo de las razones de prevalencias crudas $(R P(c))$ y ajustadas $(R P(a))$ se construyó un modelo lineal generalizado (MLG) con familia Poisson, función de enlace log y varianzas robustas (modelo 1). Así mismo, se construyó un MLG poisson log, con varianzas robustas (modelo 2) ajustado por edad, para ver el efecto que tiene el número de enfermedades o comorbilidades crónicas sobre la presencia de prostatitis aguda bacteriana en la población en estudio.

\section{Aspectos éticos}

El estudio fue revisado y aprobado para su ejecución por el Comité Institucional de Ética en Investigación (CIEI) de la Universidad Científica del Sur (código de registro: 223-2019-PRE15) y el Comité de Ética e Investigación del Hospital Militar Central (1258/AA11/8/HMC/DADCI), quienes autorizaron el análisis de los registros obtenidos del archivo de historias clínicas. Los investigadores protegieron la confidencialidad de los pacientes utilizando códigos específicos para cada una de las historias clínicas.

\section{RESULTADOS}

Un total de 142 historias clínicas fueron analizadas en el presente estudio. La población incluida presento un rango de edad entre los 52 y 89 años, con un promedio de edad de 70,2 $\pm 9,6$. La presencia PAB fue $15,5 \%$ en aquellos pacientes que previamente habían sido sometidos a biopsia prostática transrectal. La frecuencia de algunos antecedentes de enfermedades 0 comorbilidades crónicas descritos previamente en la literatura en esta población fueron diabetes $(9,9 \%)$, hipertensión arterial $(19,7 \%)$, insuficiencia renal crónica $(4,2 \%)$. 
La distribución de PAB, según algunos potenciales factores asociados descritos previamente, se reporta en la Tabla 1. En resumen, debemos mencionar que la PAB se presentó con mayor frecuencia en pacientes diabéticos $(42,9 \%)$, en pacientes con hipertensión $(28,6 \%$ y en pacientes con insuficiencia renal crónica $(66,7 \%)$, presentando en todos los casos diferencias significativas $(p<0,05)$.

Tabla 1. Factores asociados a la presencia de prostatitis aguda bacteriana en pacientes sometidos a biopsia prostática transrectal $(n=142)$.

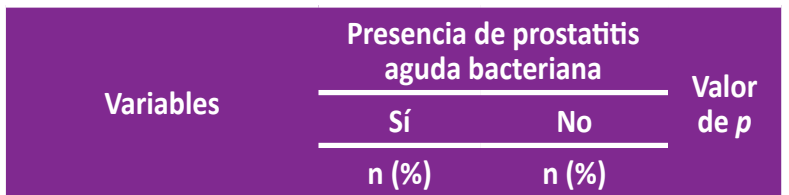

Edad en años

$0,089^{\ddagger}$

$\begin{array}{lcc}50-65 & 11(23,40) & 36(76,60) \\ 66-75 & 4(7,55) & 49(92,45) \\ >75 & 7(16,67) & 35(83,33)\end{array}$

Diabetes tipo 2

$0,009^{\dagger}$

$\begin{array}{lcc}\text { No } & 16(12,50) & 112(87,50) \\ \text { Sí } & 6(42,86) & 8(57,14)\end{array}$

Hipertensión arterial

No $\quad 14(12,28) \quad 100(87,72)$

Sí $\quad 8(28,57) \quad 20(71,43)$

Insuficiencia renal

$\begin{array}{lll}\text { No } & 18(13,24) & 118(86,76) \\ \text { Sí } & 4(66,67) & 2(33,33)\end{array}$

Accidente cerebrovascular

$\begin{array}{lcc}\text { No } & 20(14,71) & 116(85,29) \\ \text { Sí } & 2(33,33) & 4(66,67)\end{array}$

Infarto agudo al miocardio

$\begin{array}{lcc}\text { No } & 22(15,71) & 118(84,29) \\ \text { Sí } & 0(0,00) & 2(100,00)\end{array}$

Prostatitis crónica

$\begin{array}{lcc}\text { No } & 20(14,49) & 118(85,51) \\ \text { Sí } & 2(50,00) & 2(50,00)\end{array}$

Otros factores*

$0,001^{\ddagger}$

\begin{tabular}{lll} 
No & $12(10,53)$ & $102(89,47)$ \\
Sí & $10(35,71)$ & $18(64,29)$ \\
\hline
\end{tabular}

* Otros factores incluyen: glaucoma, artrosis, hipotiroidismo, gastritis

$\uparrow$ Prueba exacta de Fisher

₹ Prueba de Chi cuadrado de Pearson

El modelo de regresión ajustado se construyó con aquellas variables que fueron significativas en el análisis bivariado e incluyo a la edad, la cual es una variable confusora por defecto y considerada así en diferentes estudios en el tópico. Se pudo observar que la probabilidad de presentar PAB en aquellos sujetos de 66 - 75 años fue del $80 \%$, menor en comparación con aquellos de 50-65 años ( $p<0,011)$. El antecedente de diabetes mellitus tipo 2 (RPa: 3,18), la hipertensión arterial (RPa: 1,78), la insuficiencia renal ( $\mathrm{RPa}: 2,73$ ) y la prostatitis crónica ( $\mathrm{RPa}: 4,5)$ fueron factores que se describen en la literatura como asociados con el riesgo de desarrollar PAB. Sin embargo, en nuestra muestra y modelo final no se encontraron asociados de forma significativa (Tabla 2).

Finalmente, el modelo que evaluó la presencia del número de antecedentes de enfermedad o comorbilidades crónicas (Tabla 3) muestra que aquellos sujetos de 66-75 años tuvieron 50\% menor probabilidad de presentar PAB que aquellos sujetos de 50-65 años, y que los sujetos mayores de 75 años tenían 2,7 veces mayor probabilidad de presentar PAB que el grupo más joven. Así mismo, se observó que a medida que un sujeto presentaba mayor número de comorbilidades, la probabilidad de presentar una $\mathrm{PAB}$ se incrementaba con el número de enfermedades, siendo 4,8 veces mayor en aquellos con una enfermedad $(p<0,001), 5,8$ con dos enfermedades $(p=0,046)$ y 10,5 veces mayor en aquellos con tres enfermedades $(p=0,006)$ en comparación con el grupo que no tenía antecedentes de enfermedades crónicas.

\section{DISCUSIÓN}

La PBA, que se produce luego de una biopsia prostática, ocurre debido a que los microorganismos penetran el tejido mediante la inoculación directa de bacterias de la mucosa rectal a través de la aguja de biopsia ${ }^{[2]}$. Si bien las complicaciones después de una biopsia transrectal son comunes, las complicaciones infecciosas son algunas de las más graves que enfrenta esta población de pacientes ${ }^{[11]}$. Batura y Gopal Rao ${ }^{[12]}$ informaron que aproximadamente que entre el $2,15 \%$ y el $3,6 \%$ de los pacientes que se sometieron a biopsia transrectal en Inglaterra y Gales fueron readmitidos por complicaciones infecciosas.

La mortalidad, posterior a una biopsia transrectal, es extremadamente rara, y la mayoría de las muertes reportadas son causadas por shock séptico. Simsir et al., mostraron que existe una probabilidad de 3,06\% de sepsis después de una biopsia prostática transrectal ${ }^{[13]}$. Wagenlehner et al. ${ }^{[14]}$ informaron que $3,5 \%$ de los pacientes tenían infecciones urinarias febriles y el $3,1 \%$ requirió hospitalización después del procedimiento.

Simsir et al. ${ }^{[13]}$ y Carignan et al. ${ }^{[15]}$ reportaron que la enfermedad pulmonar obstructiva crónica, la diabetes, la hiperplasia benigna de próstata, la presencia de catéter y el haber tenido una hospitalización reciente son factores de riesgo de complicaciones infecciosas. En nuestro estudio la frecuencia de PBA como complicación luego de una biopsia transrectal fue de 15,5\%. En lo que respecta a la variable edad, se vio que el desarrollo de la PBA se dio con mayor frecuencia en el rango de edad de 50-65 años, luego presentó una disminución en los pacientes de 66-75 y volvió a presentar un aumento en los pacientes mayores de 75 años. Esta presentación de distribución de la enfermedad según edad también fue reportada por Coker y Dierfeldt ${ }^{[1]}$. 
Tabla 2. Modelo de regresión crudo y ajustado de factores asociados a prostatitis aguda bacteriana en pacientes sometidos a biopsia prostática transrectal $(n=142)$.

\begin{tabular}{|c|c|c|c|c|c|c|}
\hline \multirow{2}{*}{ Factores asociados } & \multicolumn{3}{|c|}{ Modelo bivariado } & \multicolumn{3}{|c|}{ Modelo multivariado } \\
\hline & $\mathrm{RP}(\mathrm{c})$ & IC 95\% & Valor de $p$ & $\mathrm{RP}(\mathrm{a})$ & IC $95 \%$ & Valor de $p$ \\
\hline \multicolumn{7}{|l|}{ Edad en años } \\
\hline $50-65$ & Ref. & & & Ref. & & \\
\hline $66-75$ & 0,32 & $0,10-1,01$ & 0,053 & 0,20 & $0,06-0,69$ & 0,011 \\
\hline$>75$ & 0,71 & $0,28-1,84$ & 0,483 & 0,38 & $0,12-1,22$ & 0,104 \\
\hline \multicolumn{7}{|l|}{ Diabetes mellitus tipo 2} \\
\hline No & Ref. & & & Ref. & & \\
\hline Sí & 3,43 & $1,34-8,76$ & 0,010 & 3,18 & $0,92-11,03$ & 0,069 \\
\hline \multicolumn{7}{|l|}{ Hipertensión arterial } \\
\hline No & Ref. & & & Ref. & & \\
\hline Sí & 2,33 & $0,98-5,54$ & 0,057 & 1,78 & $0,53-5,98$ & 0,351 \\
\hline \multicolumn{7}{|l|}{ Insuficiencia renal } \\
\hline No & Ref. & & & Ref. & & \\
\hline Sí & 5,04 & $1,70-14,88$ & 0,003 & 2,73 & $0,60-12,46$ & 0,194 \\
\hline \multicolumn{7}{|l|}{ Prostatitis crónica } \\
\hline No & Ref. & & & Ref. & & \\
\hline Sí & 3,45 & $0,81-14,76$ & 0,095 & 4,50 & $0,99-20,43$ & 0,052 \\
\hline
\end{tabular}

$\mathrm{RP}(\mathrm{c})$ : razón de prevalencia crudo; RP(a): razón de prevalencia ajustado; IC 95\%: intervalo de confianza al 95\%; Ref.: categoría de referencia.

Tabla 3. Análisis multivariado del efecto del número de comorbilidades crónicas sobre la presencia de prostatitis aguda bacteriana en pacientes sometidos a biopsia prostática transrectal $(n=142)$.

\begin{tabular}{|c|c|c|c|c|c|}
\hline Factores & $n / N$ & $\%$ & $\mathrm{RP}(\mathrm{a})$ & IC $95 \%$ & Valor de $p$ \\
\hline \multicolumn{6}{|l|}{ Edad } \\
\hline $50-65$ & $11 / 47$ & 23,4 & Ref. & & \\
\hline $66-75$ & $4 / 53$ & 7,6 & 0,52 & $0,14-1,87$ & 0,317 \\
\hline$\geq 75$ & $7 / 42$ & 16,7 & 2,65 & $0,86-8,13$ & 0,088 \\
\hline \multicolumn{6}{|l|}{ Enfermedades crónicas } \\
\hline Ninguna & $10 / 108$ & 9,3 & Ref. & & \\
\hline 1 & $8 / 24$ & 33,3 & 4,80 & $1,86-12,42$ & 0,001 \\
\hline 2 & $2 / 6$ & 33,3 & 5,76 & $1,03-32,13$ & 0,046 \\
\hline 3 & $2 / 4$ & 50,0 & 10,47 & $1,98-55,41$ & 0,006 \\
\hline
\end{tabular}

$\mathrm{RP}(a)$ : razón de prevaleinca ajustado; IC 95\%: intervalo de confianza al 95\%; Ref.: categoría de referencia.

Los factores asociados a la presencia de PBA, fueron la presencia de diabetes, hipertensión e insuficiencia renal, los que mostraron un efecto, siendo mayor la frecuencia de PBA en aquellos sujetos que presentaron dichos factores. Sin embargo, esta asociación no fue significativa. Por otro lado, Semins et al. ${ }^{[16]}$ informó que los pacientes obesos eran más vulnerables a desarrollar una complicación infecciosa; Wu et al. ${ }^{[17]}$, reportó que la obesidad (IMC $>28 \mathrm{~kg} / \mathrm{m}^{2}$ ) fue un factor de riesgo independiente para infección post biopsia (OR: 3,383; IC 95\% 1,327-8,626). En nuestro estudio, no se presentaron pacientes obesos por lo que no se pudo evaluar su asociación. Probablemente, debido a que se ha trabajado con una muestra de una población que se ha desarrollado dentro del ámbito militar lo que los ha condicionado a mantener un peso adecuado.
En lo que respecta a la diabetes, en nuestro estudio, la PAB se presentó con mayor frecuencia en estos pacientes que en aquellos no diabéticos $(p<0.001)$. De acuerdo con estudios previos, el antecedente de diabetes también es factor de riesgo de infección después de una biopsia de próstata. Carignan et al. ${ }^{[15]}$ reportó en un estudio de casos y controles, de un centro de atención terciaria canadiense, que la diabetes fue un factor de riesgo independiente para complicaciones infecciosas después de una biopsia de próstata. Este hallazgo también se demostró en un ensayo aleatorizado europeo realizado por Loeb et al. ${ }^{[18]}$ y Tsu et al. ${ }^{[19]}$ donde informaron que la diabetes estaba asociada con infección después de una biopsia prostática transrectal. 
La mayoría de estudios acerca de diabetes muestra que esta enfermedad incrementa el riesgo no solo de contraer infecciones sino de desarrollar complicaciones. Este riesgo incrementado en pacientes que sufren de diabetes mellitus tipo 2 se ha visto relacionado a los estados continuos de hiperglicemia y como estos deterioran los efectos de quimiotaxis y fagocitosis ${ }^{[20]}$.

Nuestro estudio presentó algunas limitaciones. Primero, el pequeño número de sujetos no permitió que los factores evaluados sean significativos en los análisis multivariados, lo cual afecto directamente el poder estadístico del estudio; sin embargo, consideramos que las medidas de efecto encontradas son clínicamente relevantes. Segundo, este fue un estudio retrospectivo que utilizó datos derivados de un solo centro en un periodo de 5 años, incluyéndose únicamente las historias clínicas con datos completos; por lo tanto, no podemos extrapolar nuestros resultados a toda la población o grupos semejantes. Consideramos que es necesario ejecutar un estudio prospectivo con un mayor número de sujetos y un adecuado seguimiento y monitoreo a nivel multicéntrico para confirmar los resultados de nuestro estudio. En tercer lugar, existe la posibilidad de que los sujetos que hayan presentado síntomas clínicos de PBA hayan acudido a otros nosocomios, por lo que habría un sesgo en los resultados finales. En cuarto lugar, nuestra población no cuenta con un resultado de urocultivo negativo previo al procedimiento por lo que no se puede asegurar que los pacientes que presentaron PBA no hayan contraído la infección antes de la realización de la biopsia.

Concluimos que la frecuencia de PAB, como una complicación luego de una biopsia transrectal, fue mayor al 15\%. El antecedente de diabetes mellitus tipo 2 y el número de comorbilidades crónicas incrementan la probabilidad de presentar PAB. Se recomienda realizar un mayor número de estudios y cohortes de seguimiento que permitan verificar nuestros resultados y entender mejor los factores de riesgo para desarrollar prostatitis aguda bacteriana.

Contribuciones de autoría: Todos los autores formularon y diseñaron la investigación. SCM y TLL recolectaron los datos. SAM Superviso la ejecución de la investigación. JMQ realizó los análisis estadísticos. Todos los autores redactaron y aprobaron la versión final del manuscrito

Potenciales conflictos de interés: Los autores declaran no tener algún tipo de conflicto de intereses.

Fuente de financiamiento: autofinanciado.

\section{ORCID:}

Stefanie Collado-Mendiola, https://orcid.org/0000-0002-8903-1393 Tiziana Lenz-Lee, https://orcid.org/0000-0001-9586-3535 Sergio D. Alban-Moral, https://orcid.org/0000-0002-2409-6559 Jorge L. Maguiña, https://orcid.org/0000-0002-4136-7795

\section{REFERENCIAS BIBLIOGRÁFICAS}

1. Coker TJ, Dierfeldt DM. Acute bacterial prostatitis: diagnosis and management. Am Fam Physician. 2016;93(2):114-20.

2. Kyriazis ID, Georgiopoulos I, Liatsikos EN. (2014) Prostatitis. In: Merseburger A, Kuczyk M, Moul J (eds). Urology at a Glance. Berlin, Heidelberg: Springer; 2014. p. 245-8. doi: 10.1007/978-3-642-548598_46

3. Ramakrishnan K, Salinas RC. Prostatitis: acute and chronic. Prim Care: Clinics in Office Practice. 2010;37(3):547-63. doi: 10.1016/j. pop.2010.04.007

4. Matlaga BR, Eskew LA, McCullough DL. Prostate biopsy: Indications and technique. J Urol. 2003;169(1):12-9. doi: 10.1016/50022-5347(05)64024-

5. Vélez D, Vicens A, Ozonas M. Profilaxis antibiótica en la biopsia transrectal de próstata. Actas Urol Esp. 2009;33(8):853-9.

6. Zani LE, Clark AO, Rodrigues Netto Jr, N. Antibiotic prophylaxis for transrectal prostate biopsy [Systematic Review]. Cochrane Database Syst Rev. 2011;5(5):5.

7. Seo, $Y$, Lee $G$. New bacterial infection in the prostate after transrectal prostate biopsy. J Korean Med Sci. 2018;33(17):1-10. doi: 10.3346/ jkms.2018.33.e126

8. Repetto E. Relación de prostatitis en la aparición del cáncer de próstata y la hiperplasia benigna prostática. Revista Cubana de Urología [Internet]. 2019 [citado el 27 de mayo de 2019];8(1):[aprox. 11 p.]. Disponible en: http://www.revurologia.sld.cu/index.php/rcu/article/view/502

9. Kim SJ, Kim SI, Ahn HS, Choi JB, Kim YS, Kim SJ. Risk Factors for Acute Prostatitis after Transrectal Biopsy of the Prostate. Korean J Urol. 2010;51(6):426-30. doi: 10.4111/kju.2010.51.6.426

10. Dean AG, Sullivan KM, Soe MM. OpenEpi: Open Source Epidemiologic Statistics for Public Health, Version 2.3.1 [Internet]. Updated 2013/04/06, accessed 2020/01/04. Disponible en: www.OpenEpi.com

11. Wu YP, Li XD, Ke ZB, Chen SH, Chen PZ, Wei Y, et al. Risk factors for infectious complications following transrectal ultrasound-guided prostate biopsy. Infect Drug Resist. 2018;11:1491-7. doi: 10.2147/IDR.S171162

12. Batura D, Gopal Rao $G$. The national burden of infections after prostate biopsy in England and Wales: a wake-up call for better prevention. J Antimicrob Chemother. 2013;68(2):247-9.

13. Simsir A, Kismali E, Mammadov R, Gunaydin G, Cal C. Is it possible to predict sepsis, the most serious complication in prostate biopsy? Urol Int. 2010;84(4):395-9.

14. Wagenlehner FM, van Oostrum E, Tenke P, Tandogdu Z, Çek M, Grabe $M$, et al. Infective com- plications after prostate biopsy: outcome of the Global Prevalence Study of Infections in Urology (GPIU) 2010 and 2011 a prospec- tive multinational multicentre prostate biopsy study. Eur Urol. 2013;63(3):521-7.

15. Carignan A, Roussy JF, Lapointe V, Valiquette L, Sabbagh R, Pépin J. Increasing risk of infectious complications after transrectal ultrasoundguided prostate biopsies: time to reassess antimicrobial prophylaxis? Eur Urol. 2012;62(3):453-9.

16. Semins MJ, Shore AD, Makary MA, Weiner J, Matlaga BR. The impact of obesity on urinary tract infection risk. Urology. 2012;79(2):266-9.

17. Wu X, Yu C, Li T, Lin L, Xu Q, Zhu Q, et al. Obesity was an independent risk factor for febrile infection after prostate biopsy: A 10-year single center study in South China. Medicine (Baltimore). 2018;97(1):e9549. doi: 10.1097/MD.0000000000009549

18. Loeb S, van den Heuvel S, Zhu X, Bangma CH, Schröder FH, Roobol MJ. Infectious complications and hospital admissions after prostate biopsy in a European randomized trial. Eur Urol. 2012;61(6):1110-4.

19. Tsu JH, Ma WK, Chan WK, Lam BH, To KC, To WK, et al. Prevalence and predictive factors of harboring fluoroquinolone-resistant and extendedspectrum $\beta$-lactamase- producing rectal flora in Hong Kong Chinese men undergoing transrectal ultrasound-guided prostate biopsy. Urology. 2015;85(1):15-22. doi: 10.1016/j.urology.2014.07.078

20. Kanafani ZA, Kourany WM, Fowler VG, Levine DP, Vigliani GA, Campion $\mathrm{M}$, et al. Clinical characteristics and outcomes of diabetic patients with Staphylococcus aureus bacteremia and endocarditis. Eur J Clin Microbio Infect Dis. 2009;28(12):1477-82. doi: 10.1007/s10096-009-0808-3 